\title{
Accretion onto Pre-Main Sequence Binary Stars: The Circumstellar Environment
}

\author{
Robert D. Mathieu \\ Department of Astronomy, University of Wisconsin, Madison WI 53706
}

\begin{abstract}
Observations show that mass accretion can occur at the surfaces of stars in young binaries of all separations. Given that resonant torques have been expected to clear gaps and dam the inward flow of material from circumbinary disks, this result begs the question of how mass accretion is supplied in binary systems. In this paper we address this question in the context of close binaries. Spectral energy distributions indicate that dynamical clearing is not complete for close binaries having massive circumbinary disks. High-resolution infrared spectra of $\mathrm{CO}$ rotational and ro-vibrational lines have also detected small amounts of gas which can be located in the region of dynamical clearing. Thus we have signatures of matter from the circumbinary environment through the predicted gap region to the stellar surfaces. We suggest that accretion streams may supply both dynamically cleared regions ánd circumstellar disks.
\end{abstract}

\section{Introduction}

The observation of mass accretion diagnostics in classical $\mathrm{T}$ Tauri binaries of all separations indicates that accretion continues at the stellar surfaces. However, dynamical theory has predicted that the balance of viscous and resonant forces at the inner edge of a circumbinary disk would prevent any flow of circumbinary material across the gap. As such, the source of the accreting material is an outstanding issue in our understanding of binary-disk interactions and ultimately in the binary formation process itself.

Presumably the accreting material flows onto the stellar surfaces from circumstellar disks. The consequence of continued accretion at the stellar surfaces would thus ultimately be exhaustion of the circumstellar disks and the cessation of accretion. As such, one might expect the lifetime of accretion for binaries to be shorter than for single stars, and indeed to differ between close and wide binaries. In fact there is little observational evidence in support of these expectations. Prato \& Simon (1995) find no difference in the frequency of accretion diagnostics between single and binary stars. Active accretion in wide binaries, with separations several times greater than typical disk radii ( $\mathrm{a}>>100 \mathrm{AU})$, is not a surprise; here the circumstellar disks are largely unaffected by their distant companions and thus are likely to have accretion histories similar to single stars. More surprising, perhaps, is a correlation between the presence of accretion onto primary and secondary stars, as reviewed by Prato and Monin in this volume. 


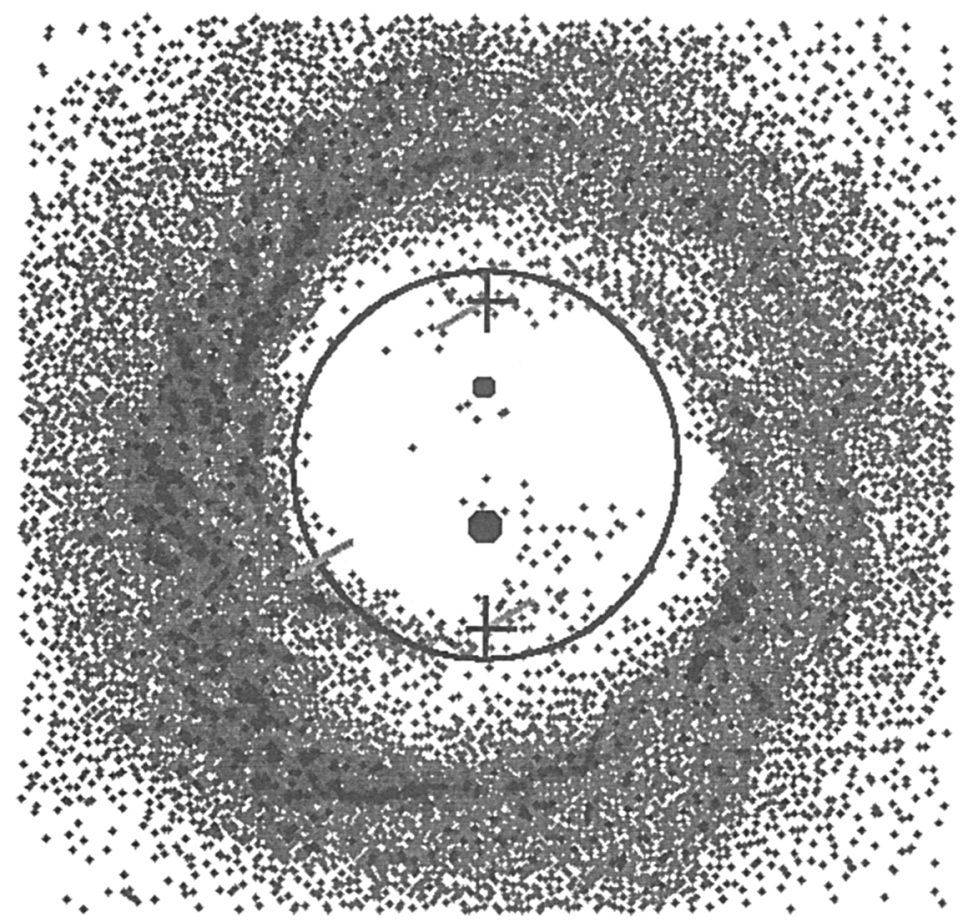

Figure 1. SPH simulation of a binary system $(\mathrm{e}=0.5, \mathrm{q}=0.9)$ surrounded by a circumbinary disk. Material flows from the circumbinary disk to the binary in two quasi-spiral accretion streams. From Artymowicz \& Lubow 1996.

Perhaps most remarkably, the observational diagnostics for accretion are present among even the very closest binary stars. As one considers binaries of smaller and smaller separation, eventually a separation must be reached where the circumstellar disk radii are so small as to not permit circumstellar masses adequate to supply the observed accretion rate for any substantial duration. And yet binaries showing accretion diagnostics are found for separations of order 10 $\mathrm{AU}$ (DF Tau), $1 \mathrm{AU}$ (GW Ori), and even 0.1 AU (UZ Tau E). These binaries show evidence for massive circumbinary disks, and there can be little doubt that the material accreting at the stellar surfaces must derive from these disks.

The essential question, then, is how the circumbinary material flows from the circumbinary disk to the surfaces of the stars. Clearly, the environment of the binary must be a more dynamically complex domain than previously thought. One recent suggestion has been the presence of accretion streams from the circumbinary disk to the circumstellar disks. Artymowicz \& Lubow (1996) have found in SPH simulations that for sufficiently warm and viscous circumbinary disks, material can flow in such streams at mass transfer rates equivalent to those expected from continuous disks around single stars. An example of such an accretion stream in an SPH simulation is shown in Figure 1. 
This paper describes several classical $\mathrm{T}$ Tauri binaries that challenge the classical picture of dynamically cleared gaps with dammed circumbinary disks. It then presents a new approach to probing the circumstellar environments of binaries with infrared emission lines, finding that indeed the gaps still contain small amounts of gas that may feed the ongoing accretion.

\section{Case Studies: Challenges for Disk-Clearing Theory}

The past decade saw the first attempts to study disk structure around young binary stars. Perhaps the most remarkable results have been obtained for the binary GG Tau, as reviewed by several papers in this volume. This 40 AU binary resides within the cleared hole of a circumbinary ring, and represents strong evidence for dynamical clearing. The binary still shows accretion diagnostics and circumstellar disks, but the presence of material or streams within the cleared region remains to be seen. Images of UY Aur show similar structures.

More indirect studies of disk clearing have been made through analysis of spectral energy distributions (SEDs). The essential idea is that cleared regions remove emitting material of a characteristic temperature, thereby leading to a deficit of emission at corresponding wavelengths. First applied to GW Ori (Mathieu, Adams \& Latham 1991), an extensive SED study has been done by Jensen \& Mathieu (1997). They found several short-period binaries whose SEDs indicated dynamical clearing - but at the same time they found that the classical $\mathrm{T}$ Tauri binary AK Sco showed no evidence for clearing in its SED (Jensen, this volume). AK Sco turns out not to be an isolated case - here we describe two other binaries which present challenges to the dynamical theory of disk clearing.

\subsection{Challenge 1: UZ Tau E}

After T Tau itself, UZ Tau $\mathrm{E}$ is arguably the most classical of all $\mathrm{T}$ Tauri stars. Its properties include: high-amplitude, irregular photometric variability; $\mathrm{H} \alpha$ emission equivalent width in excess of $100 \AA$; strong ultraviolet excess; very heavily veiled spectra; and large infrared excesses. Indeed, it has been classified as an eruptive variable with behavior akin to FU Ori stars (Herbig 1977). All of these phenomena are now associated with accretion disks actively transferring matter from the disk onto the surface of the star. In this context, observations have shown UZ Tau E to be surrounded by a massive $\left(\approx 0.6 M_{\odot}\right)$ disk with a large accretion rate of order $10^{-6} M_{\odot} y r^{-1}$ (Hartigan, Edwards \& Ghandour 1995; Jensen, Koerner \& Mathieu 1996). Thus, all of the observational data suggest a single star surrounded by an actively accreting disk which is continuous from very near the stellar surface to its outer radius of several hundred AU.

All, that is, except one set of observations: multiple radial-velocity measurements show UZ Tau E to be a binary star with an orbital period of 19.1 days and an eccentricity of 0.3 (Mathieu, Martin \& Maguzzu 1996). Only the primary star has been detected spectroscopically and so the dimensions of the orbit are not certain. But for an estimated primary mass of $0.2 M_{\odot}$ and a unit mass ratio, the periastron separation is only $0.07 \mathrm{AU}$. For a stellar radius of 3 $R_{\odot}$, this periastron separation is only 5 stellar radii. 
Thus, despite all indications that UZ Tau E is the archetype of a single star and accretion disk, in fact there is a binary star acting like an eggbeater ${ }^{1}$ in the inner core of the disk. According to classical dynamical theory, this binary should have cleared a gap (actually, more a central hole in this case) in the disk with an outer radius of 0.3 AU. Very small circumstellar disks are permitted around each star, but clearly these disks cannot sustain the observed accretion rate without being replenished. Furthermore the hole cannot be completely empty of matter. The infrared SED of UZ Tau E is well represented by a power law; the SED does not show a deficiency of emission at the shortest wavelengths (Jensen et al. 1996). However, the amount of matter required to provide such a power-law energy distribution is very small (e.g., Jensen \& Mathieu 1997).

In short, if our interpretation of accretion diagnostics are correct, then in UZ Tau $\mathrm{E}$ we find a close binary with an ample reservoir of mass in its circumbinary disk, a large accretion rate at the stellar surfaces, and evidence for at least some material in the vicinity of the dynamically predicted gap. This picture demands an accretion flow from the circumbinary disk to the stellar surfaces, perhaps with a brief interlude in circumstellar disks.

\subsection{Challenge 2: DQ Tau}

The T Tauri star DQ Tau is in many ways a twin of UZ Tau E, except that all of the disk and accretion diagnostics are reduced in magnitude. DQ Tau is a photometric variable, occasionally shows $\mathrm{H} \alpha$ emission equivalent widths in excess of $100 \AA$, and has a moderately veiled spectrum. Accretion rate estimates range from $10^{-8} M_{\odot} y r^{-1}$ to less than $10^{-9} M_{\odot} y r^{-1}$ (Hartigan et al. 1995, Gullbring et al. 1998). DQ Tau shows a power-law infrared SED, and has a moderately massive circumbinary disk (0.002 - $0.02 M_{\odot}$; Mathieu et al. 1997).

DQ Tau also is a close binary (Mathieu et al. 1997). Having a period of 15.8 days, an eccentricity of 0.56 , and a mass ratio of 1.0 , the orbital geometry is remarkably similar to UZ Tau E. The periastron separation is $0.06 \mathrm{AU}$ (or $13 R_{\odot}$ ) and the apastron separation is $0.4 \mathrm{AU}$. Again the binary is expected to dynamically clear a hole in the circumbinary disk, through which material must flow to support the observed mass accretion at the stellar surface. And once again, the power-law infrared SED suggests that such a gap, if present, retains at least a small amount $\left(\approx 5 x 10^{-10} M_{\odot}\right)$ of matter. But DQ Tau provides a critical additional clue, for all of the accretion diagnostics are periodic.

The first evidence for periodic accretion was provided by broadband photometric monitoring. The light curves in VRI are shown in Figure 2 of Stassun (this volume). DQ Tau was seen to have repeated brightening events which were brief in duration. Time series analyses showed these events to be periodic with a period of $15.8^{d}$ - identical to the binary orbital period. More specifically, the brightenings are phased with periastron passage of the stars.

During the brightenings the integrated light of the system becomes bluer, suggesting an accretion origin. The linkage to accretion became secure with spectroscopic monitoring, where both the spectral veiling and $\mathrm{H} \alpha$ emission were

\footnotetext{
${ }^{1}$ With regards to Gibor Basri.
} 
shown to also increase in phase with periastron passage (Figure 4 in Stassun; Basri, Johns-Krull \& Mathieu 1997).

The significance of this discovery lies in the contemporaneous discovery of accretion streams by Artymowicz \& Lubow (1996), as discussed in the Introduction. For a binary system very similar to DQ Tau $(\mathrm{q}=0.9$, $\mathrm{e}=0.5)$, they find a pulsed accretion stream, with the maximum accretion rate occurring at periastron passage (Figure 1). The agreement of the theoretical phasing with the periodic accretion observed in DQ Tau is very encouraging for the accretion stream mechanism. At the same time it is noteworthy that the periastron brightenings of DQ Tau have a stochastic character, with small shifts in phase and occasional periastron passages without any evident brightening.

\subsection{Summary}

Close binaries such as UZ Tau E, DQ Tau, and AK Sco present important challenges to our understanding of dynamical clearing. But they also provide critical clues for a new picture of the binary environment. Specifically, the presence of high accretion rates at stellar surfaces is typically associated with power-law SEDs which suggest that material is present throughout the immediate binary environment, including in the predicted dynamically cleared gap. Evidently dynamical clearing is not complete. The residual material in the gap may be plausibly identified as a source for the mass accretion at the stellar surfaces. Finally, in every case a circumbinary disk presents a ready reservoir to supply material in the gap. Thus in classical T Tauri close binaries, we have signatures of matter from the circumbinary environment through the predicted gap region to the stellar surfaces. In order to identify the actual process of mass flow, we need more precise probes of the inner disk structures and physical conditions.

\section{Probing the Binary Environment with Gas Emission}

Until imaging of the inner binary environments is possible - which is in the realm of upcoming optical/infrared and millimeter interferometers - only indirect probes are available. SED studies have made initial forays into these domains. However, the high emissivity of dust results in saturation of this diagnostic even with small amounts of material, and mapping the location of emitting material is dependent upon temperature distributions that are not securely understood.

Line emission from gas represents a promising alternative. Line emission provides kinematic information which allows mapping the emitting material in the context of a dynamical model (e.g., Keplerian rotation). In addition, observation of several transitions allows determination of temperatures and surface densities. Finally, gas emission can probe somewhat higher column densities than dust. Because gas temperatures within $1 \mathrm{AU}$ of the star will be a couple hundred to a couple thousand degrees, rotational and ro-vibrational transitions of molecules in the infrared are good tracers of the gas.

To explore this idea, we (J. Carr, J. Najita, and the author) have obtained high-resolution infrared spectra of DQ Tau to search for emission from the CO fundamental transitions near $4.7 \mu \mathrm{m}$. Using the CSHELL spectrograph on the NASA IRTF, we searched for and detected CO $\nu=1-0$ and $\nu=2-1$ emission. In the left panel of Figure 2 we show the $\nu=1-0 \mathrm{R}(3)$ line, flanked by two R-branch 


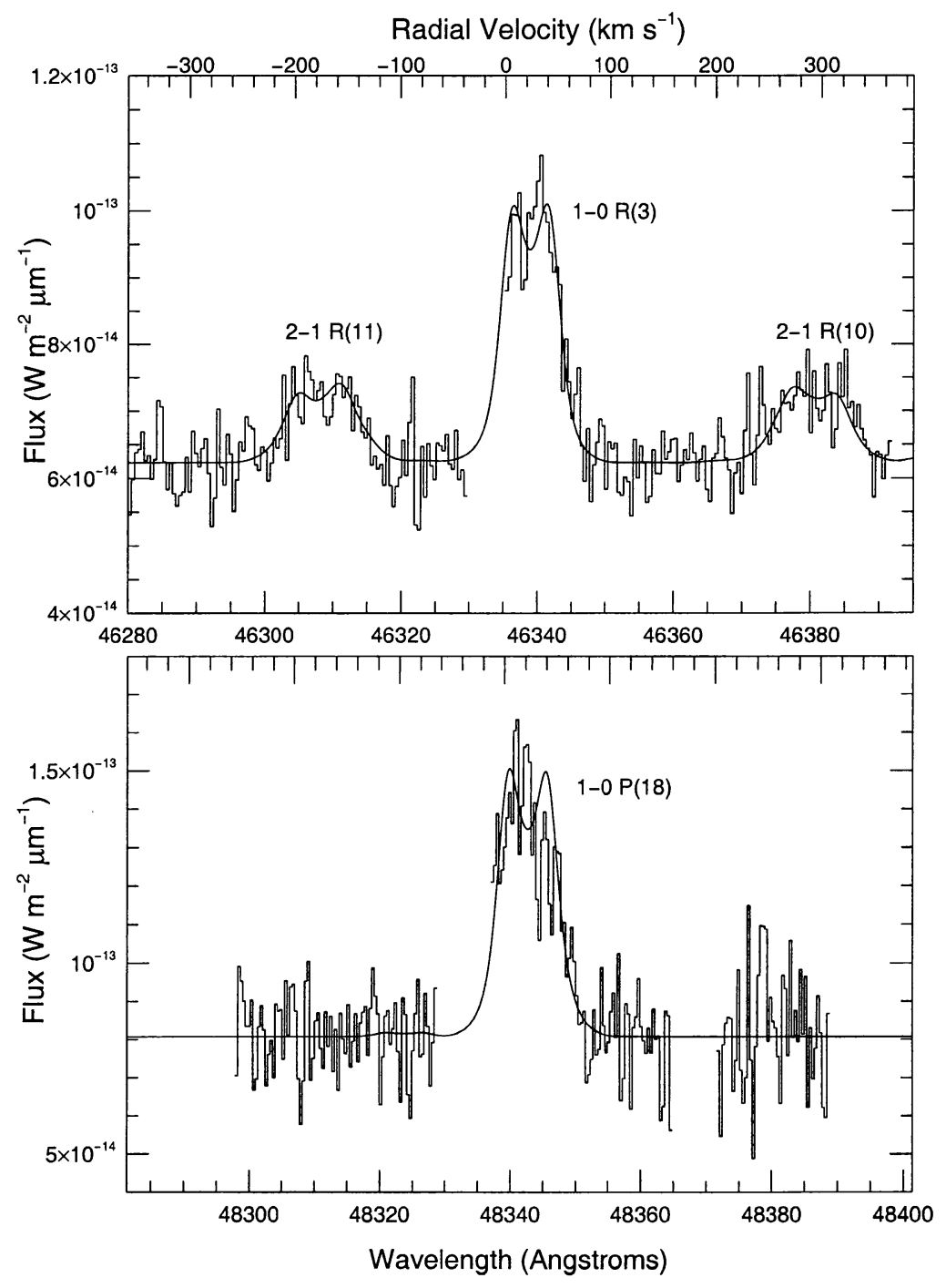

Figure 2. Infrared high-resolution spectra of the classical T Tauri binary DQ Tau. The left panel shows the CO $\nu=1-0 \mathrm{R}(3)$ line, flanked by the $C O \nu=2-1 \mathrm{R}(10)$ and $\mathrm{R}(11)$ lines. The right panel shows the CO $\nu=1-0 \mathrm{P}(18)$ line. The gap in the spectra is the core of the telluric $\mathrm{CO}$ absorption. The curve shows predicted emission from a disk with residual material in a dynamically cleared hole. The model surface density of material in the hole is $5 \times 10^{-4} \mathrm{~g} \mathrm{~cm}^{-2}$, the inner radius is < $0.1 \mathrm{AU}$, the outer radius is $0.5 \mathrm{AU}$, and the temperature at the inner edge of the circumbinary disk is $775 \mathrm{~K}$. 


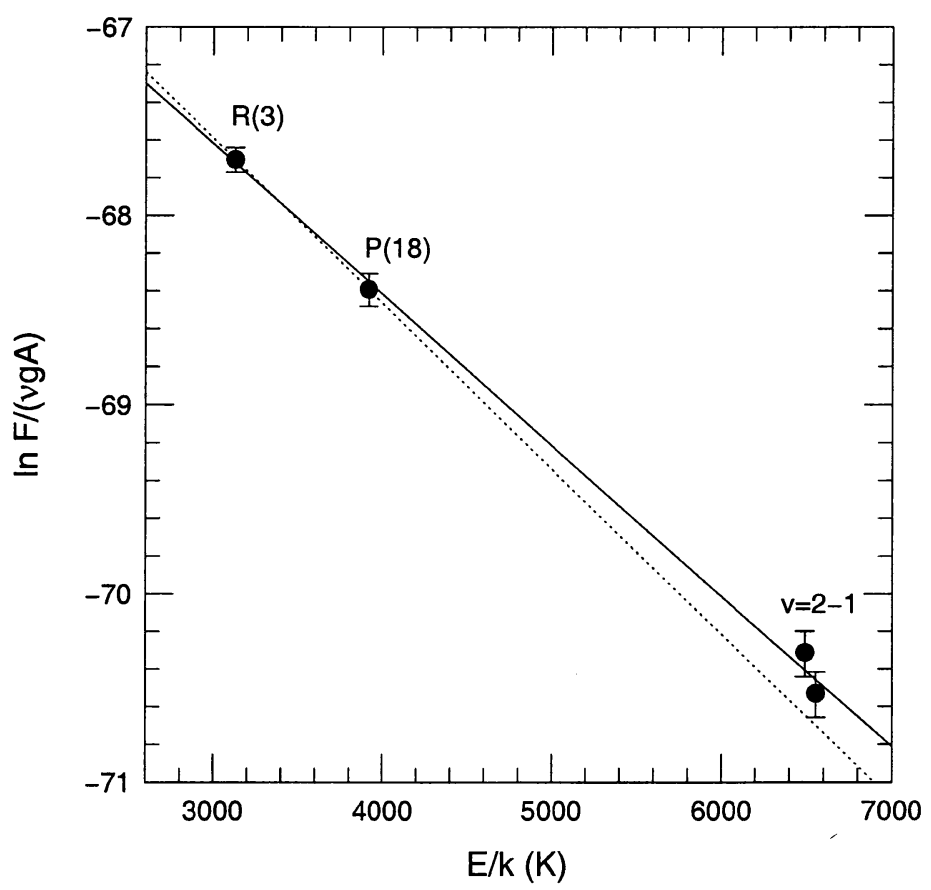

Figure 3. Excitation temperature from CO lines observed in DQ Tau. The figure shows the natural $\log$ of $F_{u l} / \nu g_{u} A_{u l}$ versus the upper energy level, where $F_{u l}$ is the line flux, $\nu$ the line frequency, $\mathrm{g}_{u}$ the statistical weight of the upper level, and $\mathrm{A}_{u l}$ the transition probability. The solid line is a fit to all four data, yielding $\mathrm{T}=1252 \pm 49 \mathrm{~K}$. The dotted line is fit to only the $\nu=1-0 \mathrm{R}(3)$ and $\mathrm{P}(18)$ lines, yielding $\mathrm{T}=1143 \pm 180$ $\mathrm{K}$.

lines of the $\nu=2-1$ transition. The right panel shows the $\nu=1-0 \mathrm{P}(18)$ line. These are the first detections of these lines from a low-mass T Tauri star (Carr, Mathieu \& Najita 2000).

The detection of line emission from three different energy levels permits determination of the excitation temperatures in the optically thin approximation. In Figure 3 we show flux as a function of energy level, appropriately scaled for the physics of each transition. A fit to all four data points yields $\mathrm{T}=1252 \pm 49$ $\mathrm{K}$. This is not significantly different from a fit to only the two $\nu=1-0$ lines, which gives $\mathrm{T}=1143 \pm 180 \mathrm{~K}$, suggesting that the rotational and vibrational levels are close to being in LTE up to at least the $\nu=2$ level. More importantly, these temperatures are suggestive of circumstellar material in the immediate vicinity of the binary. 


\section{Modelling Inner Disk Conditions}

We envision that the binary system lies at the center of an optically thick circumbinary disk. The binary has cleared a hole in this circumbinary disk, and in this hole resides optically thin dust and gas which is taken to be the origin of the emission lines. Surrounding each star may be very small, optically thick circumstellar disks.

The disk modeling procedure is similar to that described in Najita et al. (1996), except that here the line emission region is modeled as a single layer and dust opacity was included. The $\mathrm{CO}$ abundance relative to hydrogen was calculated assuming chemical equilibrium, and LTE is assumed. We take the combined binary mass for DQ Tau to be $1.3 M_{\odot}$ with a system inclination of $23^{\circ}$ (Mathieu et al. 1997). Finally, we are not in a position to model the twodimensional spatial distribution of gas flows in a binary system. Hence we model the system with an axisymmetric, Keplerian model using the combined masses of the stars. This model will be reasonably accurate near the inner edge of the circumbinary disk and near the stars. In the vicinity of the binary orbit we can only claim that the Keplerian model approximates the scale of the orbital motions.

In the modeling, the free parameters which can be varied to fit the observed lines are the radial variation of the gas temperature and surface density, and the inner and outer radii of the gap. The relative line strengths are largely determined by the gas temperature, while the absolute line fluxes are determined by the combination of gas temperature, surface density, and emitting area. The model line profile is set by the inner and outer radii of the gap, as well as by the radial dependence of the emission surface brightness (i.e., temperature and surface density).

The best-fit model is also shown in Figure 2. The spectra are not well-fit by a constant temperature model; the model shown adopts $T \approx R^{-0.5}$ and a constant surface density. The model outer radius is $0.5 \mathrm{AU} \pm 0.1 \mathrm{AU}$, at which the temperature is $775 \mathrm{~K}$; because of the low signal-to-noise the inner radius largely set by the width of the base of the line - is not well determined but is securely less than $0.1 \mathrm{AU}$. The surface density of $5 \times 10^{-4} \mathrm{~g} \mathrm{~cm}^{-2}$ represents a total mass of $4 x 10^{-11} M_{\odot}$, or only $10^{-5} M_{\oplus}$ ! Thus the observed gas emission is consistent with a dynamically cleared inner region containing a small amount of residual material.

The outer gap radius of $0.5 \mathrm{AU} \pm 0.1 \mathrm{AU}$ is consistent with the $\approx 0.4$ AU outer radius that is expected for the DQ Tau system based on the analytic calculations and smoothed particle hydrodynamic simulations of Artymowicz \& Lubow (1994) for eccentric binary systems. Furthermore the temperature derived for the gas is reasonable for material at these distances from the stars and is the same as that of the optically thin dust proposed by Mathieu et al. (1997) to reside within the gap. A connection between the gas responsible for the $\mathrm{CO}$ emission and the dust producing the near- to mid-infrared excess is also suggested by correlated brightness variations between the CO line and $4.6 \mu \mathrm{m}$ continuum strengths.

Because material must cross the gap in order to supply the on-going mass accretion onto the stars, the discovery of residual gas and dust within the gap is important. However, the relation of the emission to an accretion stream is not 
clear. Gas with a surface filling factor as small as an accretion stream would have great difficulty producing the observed emission-line fluxes. While such an optically thick stream can produce the $\mathrm{R}(1)$ emission, the $\nu=2-1$ lines then also become optically thick and too luminous.

Alternatively, an accretion rate of $\approx 10^{-8} M_{\odot} y r^{-1}$ and a dynamical time for the streaming material comparable to the binary orbital period imply a stream mass of $5 \times 10^{-10} M_{\odot}$. This mass is very similar to the mass in the gap proposed by Mathieu et al. (1997) based on the SED. However, it is an order of magnitude larger than the mass derived from gas. Again, the emission from such a stream would dominate the observed emission.

Finally, the dust associated with the derived gas mass is not sufficent to provide the observed near-infrared continuum. Interestingly, all of these difficulties would be resolved if our estimate of $\mathrm{CO}$ emissivity per gram is too high by at least an order of magnitude. At this early stage of study of the gas, such an uncertainty is possible.

Pending resolution of the $\mathrm{CO}$ emissivity question, we suggest that most of the observed $\mathrm{CO}$ emission derives from material distributed throughout the gap. Such a dispersed component would be consistent with a low-level of constant accretion that is seen at all orbital phases (Basri et al. 1997), and could be easily supplied by an accretion stream with a mass of order that inferred from the accretion rate.

\section{Conclusion}

A primary goal of this paper is to bring to the fore the fact that while we can be reasonably confident that mass accretion occurs at the surfaces of stars in binary systems, we do not have a complete understanding of how the accreting material is supplied. The problem can be divided into several domains distinguished by binary separation.

- Wide binaries (e.g., a $\gtrsim 10,000 \mathrm{AU}$ ) - With separations comparable to the size of dense molecular cores and orbital periods comparable to infall times, each star in these very wide binaries likely goes through more or less independent formation events. As such, their mass accretion evolution would differ little from single stars.

- Intermediate-separation binaries (e.g., $\mathrm{a} \approx 100$ - 10,000 AU) - Binaries in this domain have separations small compared to typical protostellar envelopes, but large compared to typical disk radii. Prato and collaborators have argued that the accretion in these systems is fed by infall from a single protostellar envelope onto both stars.

- Close binaries (e.g., a $\lesssim 100 \mathrm{AU}$ - The focus of this paper, these binaries are too close for substantial replenishment of their circumstellar disks by infall. The ubiquitous association of circumbinary disks with accretion diagnostics suggests that the circumbinary disks are the reservoirs for the mass accretion. These binaries should motivate theoretical and observational study of matter flow in the immediate environments of binary stars.

Both power-law spectral energy distributions and the detection of $1200 \mathrm{~K}$ $\mathrm{CO}$ fundamental emission are consistent with dynamically cleared gaps still having small amounts of gas and dust within them. The orbits of such material are 
unstable, suggesting that the duration of any particular bit of matter in the gap is short. While the origin of material in the gap is not yet clear, its presence is intriguing in the context of previously suggested accretion streams from the circumbinary disk to the stars. More generally, in classical T Tauri close binaries, we now have signatures of matter from the circumbinary environment through the predicted gap region to the stars. Our hypothesis here is that these signatures are in fact linked to a continuous flow of matter from the circumbinary disks to the stellar surfaces. Testing this hypothesis, and if found true, determining the physical processes by which this flow occurs, is the challenge before us.

Acknowledgments. I am very grateful to the many collaborators who have contributed so substantially to the work discussed here, especially my students Eric Jensen and Keivan Stassun. I would like to thank John Carr and Joan Najita for introducing me to the world of infrared spectroscopy and for applying their expertise to the problem of young binaries. This work was supported by the National Science Foundation through AST94-941715, and by the NASA IRTF.

\section{References}

Artymowicz, P., \& Lubow, S. H. 1994, ApJ, 421, 651

Artymowicz, P., \& Lubow, S. H. 1996, ApJ, 467, L77

Basri, G., Johns-Krull, C. M., \& Mathieu, R. D. 1997, AJ, 114, 781

Carr, J. S., Mathieu, R. D., \& Najita, J. R. 2000, AJ, submitted

Gullbring, E., Hartmann, L., Briceno, C., \& Calvet, N. 1998, ApJ, 492, 323

Hartigan, P., Edwards, S., \& Ghandour, L. 1995, ApJ, 452, 736

Herbig, G. H. 1977, ApJ, 217, 693

Jensen, E. L. N., Koerner, D. W., \& Mathieu, R. D. 1996, AJ, 111, 2431

Jensen, E. L. N., \& Mathieu, R. D. 1997, AJ, 114, 301

Mathieu, R. D., Adams, F. C., \& Latham, D. W. 1991, AJ, 101, 2184

Mathieu, R. D., Martin, E., \& Maguzzu, A. 1996, BAAS, 188, 60.05

Mathieu, R. D., Stassun, K., Basri, G., Johns-Krull, C. M., Valenti, J., Jensen, E. L. N., \& Hartmann, L. W. 1997, AJ, 113, 1841

Najita, J., Carr, J. S., Glassgold, A. E., Shu, F. H., \& Tokunaga, A. T. 1996, ApJ, 462, 919

Valenti, J. A., Basri, G., \& Johns, C. M. 1993, AJ, 106, 2024 